\title{
Altered Metabolic Profile of Triglyceride-Rich Lipoproteins in Gut-Lymph of Rodent Models of Sepsis and Gut Ischemia-Reperfusion Injury
}

\author{
Jiwon Hong ${ }^{1,2}$ (D) Shorena Nachkebia ${ }^{2} \cdot$ Soe Min Tun ${ }^{2} \cdot$ Amorita Petzer $^{1} \cdot$ John A. Windsor $^{2} \cdot$ Anthony J. Hickey $^{1}$. \\ Anthony R. Phillips ${ }^{1,2}$
}

Received: 10 June 2018 / Accepted: 29 August 2018 / Published online: 4 September 2018

(c) Springer Science+Business Media, LLC, part of Springer Nature 2018

\begin{abstract}
Background Triglyceride-rich lipoproteins are important in dietary lipid absorption and subsequent energy distribution in the body. Their importance in the gut-lymph may have been overlooked in sepsis, the most common cause of critical illness, and in gut ischemia-reperfusion injury, a common feature of many critical illnesses.

Aims We aimed to undertake an exploratory study of triglyceride-rich lipoprotein fractions in gut-lymph using untargeted metabolic profiling to identify altered metabolites in sepsis or gut ischemia-reperfusion.

Methods The gut-lymph was collected from rodent sham, sepsis, and gut ischemia-reperfusion models. The triglyceriderich lipoprotein-enriched fractions isolated from the gut-lymph were subjected to a dual metabolomics analysis approach: non-polar metabolite analysis by ultra-high performance liquid chromatography-mass spectrometry and polar metabolite analysis by gas chromatography-mass spectrometry.

Results The metabolite analysis of gut-lymph triglyceride-rich lipoprotein fractions revealed a significant increase (FDRadjusted $P$ value $<0.05)$ in myo-inositol in the sepsis group and monoacylglycerols [(18:1) and (18:2)] in gut ischemiareperfusion. There were no significantly increased specific metabolites in the lipoprotein-enriched fractions of both sepsis and gut ischemia-reperfusion. In contrast, there was a widespread decrease in multiple lipid species in sepsis (35 out of 190; adjusted $P<0.05)$, but not in the gut ischemia-reperfusion.

Conclusions Increased levels of myo-inositol and monoacylglycerols, and decreased multiple lipid species in the gut-lymph triglyceride-rich lipoprotein fraction could be candidates for new biomarkers and/or involved in the progression of sepsis and gut ischemia-reperfusion pathobiology.
\end{abstract}

Keywords Metabolomics $\cdot$ Lipoproteins $\cdot$ Lymph $\cdot$ Gut $\cdot$ Critical illness

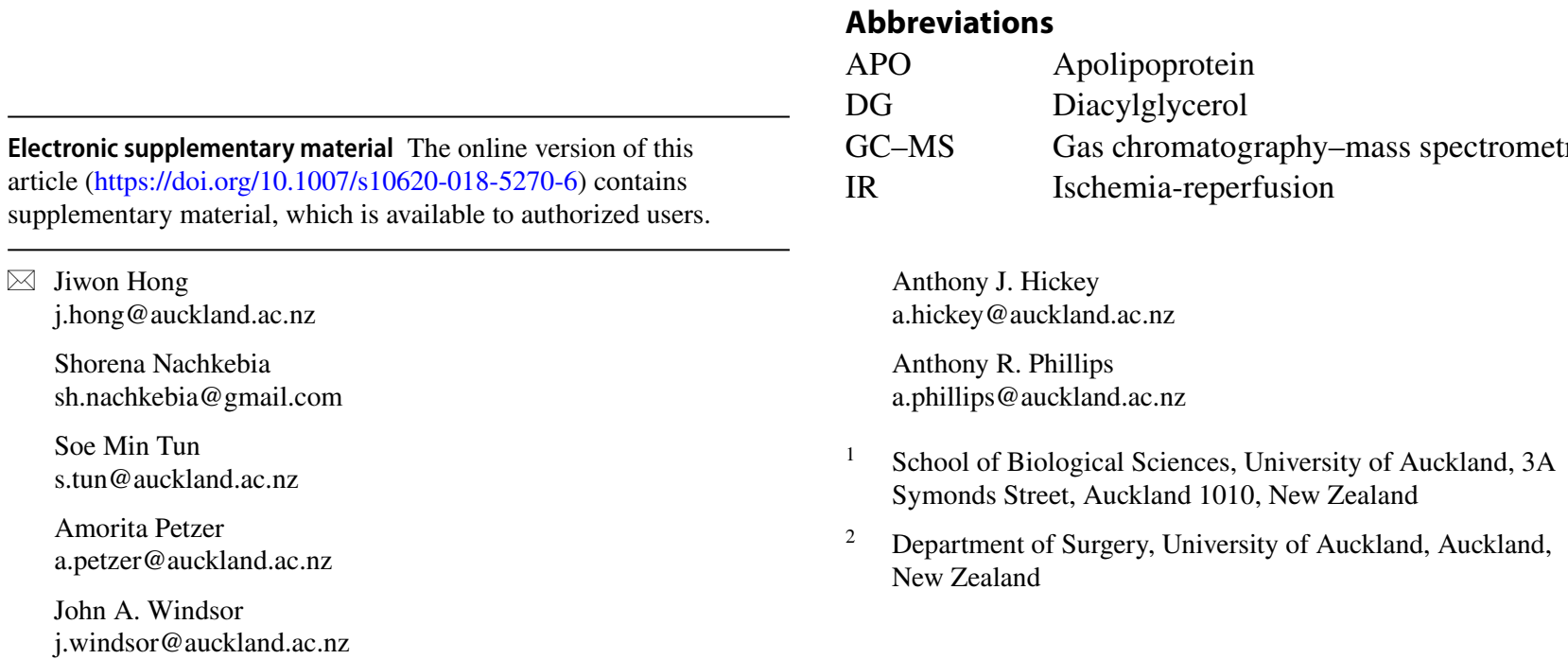




$\begin{array}{ll}\text { LPC } & \text { Lysophosphatidylcholine } \\ \text { LPE } & \text { Lysophosphatidylethanolamine } \\ \text { MG } & \text { Monoacylglycerol } \\ \text { MAP } & \text { Mean arterial pressure } \\ \text { MODS } & \text { Multiple organ dysfunction syndrome } \\ \text { PC } & \text { Phosphocholine } \\ \text { PE } & \text { Phosphoethanolamine } \\ \text { PS } & \text { Phosphoserine } \\ \text { QC } & \text { Quality control } \\ \text { TG } & \text { Triglyceride } \\ \text { TRL } & \text { Triglyceride-rich lipoprotein } \\ \text { UHPLC-MS } & \text { Ultra-high performance liquid chromatog- } \\ & \text { raphy-mass spectrometry } \\ \text { VLDL } & \text { Very low-density lipoprotein }\end{array}$

\section{Introduction}

Multiple organ dysfunction syndrome (MODS) is the progressive development of dysfunction in two or more key organs and is often seen in intensive care unit patients. MODS is the major reason of death in critically ill patients, with the mortality rate ranging between 54 and $100 \%$ depending on the number of organs affected [1]. MODS is associated with a diverse range of critical illness conditions including sepsis, acute pancreatitis, trauma, and burns. Despite this diversity, MODS typically results in a predictable sequence of organ failure, starting with lungs and heart, then followed by liver and kidneys [2]. However, the mechanisms driving MODS remain largely unknown, and the current treatment approach is predominantly generic organ support.

During critical illness, the human body maintains the perfusion of key organs by diverting blood away from the splanchnic circulation, causing a variable degree of gut ischemia-reperfusion (IR) injury and impaired gut barrier function [3]. The "gut-lymph hypothesis" [4] proposes that in critical illness, gut-derived inflammatory factors are carried in the lymph draining from the gut and transported to distant organs leading to the development of MODS. The gut-lymph is transported in the thoracic duct, which enters the bloodstream via the left neck veins immediately proximal to heart and lungs. This anatomical route of gut-lymph bypasses the potential detoxifying activity of the liver.

One of the key functions of gut-lymph is the transportation of lipids from the gut to peripheral tissues via the systemic circulation. In the normal fed state, the majority of dietary lipids are absorbed by the enterocytes in the gut, assembled into chylomicrons (the least dense class of lipoproteins), taken up into gut-lymph, and then transported to the bloodstream [5]. In the fasted state, very low-density lipoproteins (VLDLs) are the major lipoprotein class found in the gut-lymph and they transport endogenous intestinal and biliary lipids to the bloodstream [6]. These intestinal VLDLs more closely resemble chylomicrons in the gutlymph than the plasma VLDLs [7] that are consistently produced by the liver. Both chylomicrons and VLDLs in the gut-lymph have a high total lipid content (>90\% of weight) [8] and are particularly rich in triglycerides (TG; $82 \%$ and $67 \%$, respectively) [6]. These TG-rich lipoproteins (TRL) contain apolipoprotein (APO) B as their major protein component [9], and distribute energy around the body in the form of TGs. In critical illness, TG absorption by the small intestine has been reported to be markedly diminished [10], and serum TG levels are low which correlates with the severity of illness [11]. A detailed analysis of the metabolite composition of TRL in gut-lymph in critical illness has not been performed.

Metabolites are low molecular weight substrates or products in biological systems. Thousands of endogenous metabolites have been identified in the human body [12] including lipids, amino acids, organic acids, and carbohydrates. Detailed metabolite profiling provides a snapshot of metabolic state in biological systems, and this can result in the discovery of molecular mechanisms, biomarkers, and targeted treatments.

While important in lipid absorption and energy distribution, the importance of TRLs in gut-lymph may have been overlooked in critical illness. This exploratory study performed untargeted metabolic profiling on TRL-enriched fractions of gut-lymph in two rodent models of critical illness: sepsis (the most common cause of critical illness and MODS) and gut-IR (a common feature of many critical illnesses). We used a dual metabolomic analysis approach, applying ultra-high performance liquid chromatography-mass spectrometry (UHPLC-MS) to analyze nonpolar metabolites (including TG and other lipids) and the gas chromatography-mass spectrometry (GC-MS) to analyze polar metabolites (including amino acids and small sugars) to identify the changes in metabolite profiles in these critical illnesses compared with sham rodents.

\section{Methods}

\section{Rodent Models and Gut-Lymph Collection}

This animal study was approved by the University of Auckland Animal Ethics Committee. Male Sprague Dawley rats were fed ad libitum with a standard $18 \%$ protein rodent diet (Teklad 2018, Harlan Laboratories, Madison WI, USA) prior to surgery, which began for each rat at 09:00 h. General anesthesia was initiated (induction chamber; $4 \%$ isoflurane; $\mathrm{O}_{2}$ at $2 \mathrm{~L} / \mathrm{min}$ ), and then maintained with $1.5-2.5 \%$ isoflurane and a $40 \% \mathrm{O}_{2}$ /air gas ratio. The rat was placed on a warming plate to maintain its body temperature $\left(36-38{ }^{\circ} \mathrm{C}\right)$, 
which was monitored via a rectal thermometer probe. For the tracheostomy, a 14G modified angiocath (BD Medical, NJ, USA) was inserted into the trachea via a neck incision and connected to a small animal mechanical ventilator (SAR-830/P, CWE Inc., USA). Respiratory rate and peak inspiratory pressure were set at $60-80$ breaths/min and 14-18 $\mathrm{cmH}_{2} \mathrm{O}$, respectively. The end tidal $\mathrm{CO}_{2}$ was maintained at $35-45 \mathrm{ml} / \mathrm{l}$ and monitored by a capnograph (EMCO CapnoWave 3400, Respironics Inc., USA). Maintenance fluid $(0.9 \% \mathrm{NaCl})$ was administered via a right femoral vein catheter, and a $2 \mathrm{~F}$ solid-state pressure transducer (Millar Instruments Inc., TX, USA) was inserted into the right femoral artery to monitor the mean arterial pressure (MAP). A midline laparotomy was then undertaken, and the mesenteric lymph duct was cannulated with a silastic tube $(0.64 \mathrm{~mm}$ i.d, $1.1 \mathrm{~mm}$ o.d.) and secured in place with cyanoacrylate tissue glue (Aesculap, Center Valley, PA, USA).

Rats were randomized into three groups: sham, sepsis and gut-IR (4 rats per group). Sham: These control rats underwent the same intervention, but without any disease induction. Sham rats were stable throughout for the full duration of the study with a sustained MAP between 70 and $80 \mathrm{mmHg}$. Sepsis: For this model, the cecum distal to the ileocecal valve was ligated and a $0.5-\mathrm{cm}$ incision was made to release feces which was spread it to all four quadrants of the abdomen [13]. Sepsis was allowed to develop over $5 \mathrm{~h}$. Gut-IR: This was induced by use of an atraumatic Biemer clip to occlude the superior mesenteric artery for $30 \mathrm{~min}$ and then removed to allow reperfusion for $5 \mathrm{~h}$ [14].

During the $5 \mathrm{~h}$ of disease progression in both models, gut-lymph was collected on ice into tubes containing EDTA $(25 \mu \mathrm{l}$ of $40 \mathrm{mg} / \mathrm{ml}$ EDTA, per ml of gut-lymph; final $2.7 \mathrm{mM}$ ) [15]. Intraoperative MAP, heart rate, and body temperature were continuously monitored using the LabChart 5 (ADInstruments Pty Ltd, New South Wales, Australia). Maintenance fluid of $0.9 \% \mathrm{NaCl}$ was constantly infused at $18 \mathrm{ml} / \mathrm{kg}$ body weight $/ \mathrm{h}$, and additional $1 \mathrm{ml}$ fluid boluses were given to sepsis-induced rats if MAP fell below $30 \mathrm{mmHg}$. The total volume of maintenance fluid administered into each rat and the total volume of gut-lymph collected from each rat were recorded. At the end of $5 \mathrm{~h}$ gut-lymph collection, blood was collected from the inferior vena cava into $\mathrm{K}_{2} \mathrm{E}$ vacutainers (BD) and centrifuged for $10 \mathrm{~min}$ at $1900 \times g$ at $4{ }^{\circ} \mathrm{C}$. The resulting endpoint plasma was further centrifuged for $5 \mathrm{~min}$ at $16,000 \times g$ at $4{ }^{\circ} \mathrm{C}$ and stored at $-80^{\circ} \mathrm{C}$.

\section{Isolation of TRL-Enriched Fraction in Gut-Lymph}

Gut-lymph was centrifuged at $1900 \times g$ for $10 \mathrm{~min}$ at $4{ }^{\circ} \mathrm{C}$ to remove cells and debris. $3.5 \mathrm{ml}$ of gut-lymph collected from each rat was transferred into a 4.7-ml Optiseal polyallomer tube (Beckman Coulter, CA, USA), and then carefully overlaid with $1.2 \mathrm{ml}$ of saline at a density of $1.006 \mathrm{~g} / \mathrm{ml}$ that contained $0.27 \mathrm{mM}$ EDTA and approximately $0.85 \% \mathrm{NaCl}$. Lipoproteins of density $<1.006 \mathrm{~g} / \mathrm{ml}$ (chylomicrons $<0.95 \mathrm{~g} /$ $\mathrm{ml}$ and VLDLs $0.95-1.006 \mathrm{~g} / \mathrm{ml}$ [16]) were floated by ultracentrifugation at $657,000 \times g$ for $2 \mathrm{~h} 55 \mathrm{~min}$ at $10^{\circ} \mathrm{C}$ in an Optima MAX-XP ultracentrifuge using TLA-110 rotor (Beckman Coulter, CA, USA) [17]. The top 1-ml TRLenriched fraction was removed and stored at $-80{ }^{\circ} \mathrm{C}$ until metabolite analyses.

Analyte concentrations were measured in plasma, gutlymph, and TRL-enriched fractions of the gut-lymph on a Hitachi 902 Autoanalyzer (Hitachi High Technologies Corp., Tokyo, Japan) using appropriate methods including five lipid profile tests (TG, cholesterol, APOA1, APOB, and HDL-cholesterol). Specifically, these were enzymatic colorimetric (glucose, TG, and cholesterol), immunoturbidimetric (APOA1 and APOB), enzyme-linked kinetic UV (ALT and AST), kinetic UV (urea), homogenous enzymatic colorimetric (HDL), kinetic colorimetric (creatinine), and colorimetric (total protein and albumin). All the reagents were purchased from Roche (Mannheim, Germany).

One-way ANOVAs were used to analyze differences between rodent study groups for each dependent variable (i.e., rat characteristic and various analytes). The majority of variables did not require log transformation, and a post hoc Tukey's test was used to perform pairwise comparisons of the means. Some variables were log-transformed prior to analysis and confidence intervals determined on their ratios. $P$ values were adjusted for multiple testing. An adjusted $P$ value $<0.05$ was considered statistically significant. All statistical analyses were performed using the R statistical software (version 3.4.1) [18].

\section{Metabolite Extraction}

Each gut-lymph TRL-enriched sample $(200 \mu \mathrm{l})$ was extracted in $800 \mu \mathrm{l}$ of $50: 50 \mathrm{MeOH}: \mathrm{CHCl}_{3}$ containing six isotopically labeled internal standards $(0.016 \mathrm{mg} / \mathrm{ml}$ each succinic acid- $d_{4}$, glycine- $d_{5},{ }^{13} \mathrm{C}_{6}$-D-fructose, citric acid- $d_{4}$, L-alanine- $d_{7}$, and benzoic acid- $d_{5}$; Cambridge Isotopes Inc., MA, USA) using a TissueLyser II (Qiagen) set for $10 \mathrm{~min}$ at $25 \mathrm{~Hz}$, with a single 3-mm tungsten carbide bead/tube. Phase separation was achieved by adding $400 \mu \mathrm{l}$ of $\mathrm{H}_{2} \mathrm{O}$ and vortexing for $10-15 \mathrm{~s}$, followed by centrifugation at $2400 \times g$ for $15 \mathrm{~min}$. After centrifugation, a debris layer was at the interface between the upper (polar, $\mathrm{MeOH}: \mathrm{H}_{2} \mathrm{O}$ ) and lower (non-polar, $\mathrm{CHCl}_{3}$ ) phases.

For UHPLC-MS analysis, $200 \mu \mathrm{l}$ of the lower phase was carefully removed with a pipette to avoid disturbance of upper and debris layers, and gently mixed. One hundred microliters of this lower phase aliquot from each sample was pooled to make a quality control (QC) sample for nonpolar extract. Non-polar extract samples were then dried in a 
Speedvac (Savant SPD331DDA, Thermo Scientific) at $30{ }^{\circ} \mathrm{C}$ for $2.5 \mathrm{~h}$.

For GC-MS analysis, residual lower $\mathrm{CHCl}_{3}$ was removed from the extraction tube using HPLC syringe (Sigma Aldrich, MO, USA), and centrifuged at $16,000 \times g$ for $15 \mathrm{~min}$ to form a coherent pellet of debris. Two hundred microliters aliquots of MeOH: $\mathrm{H}_{2} \mathrm{O}$ supernatants were transferred to tubes containing $600 \mu \mathrm{l}$ of $\mathrm{MeOH}$ to precipitate any residual protein. A pooled QC was made by combining $200 \mu \mathrm{l}$ aliquot of each extract and gently mixing it, and $200 \mu$ of the pooled sample was dispensed into tubes containing $600 \mu \mathrm{l}$ of $\mathrm{MeOH}$. After centrifugation of both sample and QC tubes at $16,000 \times g$ for $15 \mathrm{~min}, 750 \mu \mathrm{l}$ of each was dried in a Speedvac for $16-18 \mathrm{~h}$ at $30{ }^{\circ} \mathrm{C}$ and stored at $4{ }^{\circ} \mathrm{C}$ until derivatization. Extraction blanks were prepared for both UHPLC-MS and GC-MS analyses without inclusion of TRL samples, but not included in the subsequent data analysis.

\section{UHPLC-MS Data Analysis}

Metabolites in the non-polar fraction were reconstituted in $120 \mu \mathrm{l}$ of $\mathrm{MeOH}$. UHPLC-MS analysis was performed on an Accela ultra high pressure LC system coupled to an Orbitrap Velos mass spectrometer with a heated electrospray ionization source (Thermo Fisher Scientific, Hemel Hempstead, UK). A lead-in sequence of QC injections was run first. Samples were then injected in a randomized manner in duplicate, and the pooled QC and extraction blanks were also injected. Instrumental analysis followed a previously described protocol [19]. LIPID MAPS (http://www.lipid maps.org/) was used for lipid identification and classification. A proprietary in-house approach developed at the Centre for Advanced Discovery and Experimental Therapeutics (University of Manchester, UK) was used for data analysis.

\section{GC-MS Data Analysis}

Metabolites in the polar fraction were derivatized to methoxime/trimethylsilyl (TMS) derivatives as previously described [20]. GC-MS analysis was performed using a Gerstel MPS2 autosampler (Mülheim an der Ruhr, Germany), an Agilent 7890A Gas Chromatograph with Split/Splitless inlet (Agilent; Santa Clara, CA, USA), and a Pegasus HT time-offlight mass spectrometer (LECO, Stockport, UK). The study was performed all in one batch. Samples of 3 rodent groups were run in a randomized sequence, all in duplicate, and the pooled QC and extraction blanks were also injected. A leadin sequence of $\mathrm{QC}$ injections was run at the start to condition the chromatographic system.

The "Reference Compare" method within ChromaTOF 4.5 (LECO) was used for data preparation. Three databases were employed in this study: NIST mass spectral reference library (NIST08/2008; National Institute of Standards and Technology/Environmental Protection Agency/National Institutes of Health Spectral Library, Gaithersburg, MD, USA); the Golm Metabolome Database (Max Planck Institute of Molecular Plant Physiology, Potsdam-Golm, Germany); and an in-house library developed at the University of Manchester [19]. Data reduction and metabolite abundance reporting were carried out as previously described [19].

\section{Statistical Analysis of Metabolite Data}

Kruskal-Wallis tests were used to assess significant differences in means of the metabolites between each disease and the sham group for both UHPLC-MS and GC-MS data. $P$ values were adjusted using false discovery rate (FDR) at $\alpha=0.05$, and only metabolites with an adjusted $P$ value $<0.05$ were considered statistically significant. Supplementary Data 1 show the mean and relative standard deviation of each study group; ratio of means and 95\% confidence limits for the ratios between each disease group and sham group; and the $P$ values before and after FDR adjustment. Statistical analyses were performed using the $\mathrm{R}$ statistical software (version 3.1.0).

\section{Results}

\section{General Sample Characteristics and Biochemistry}

Table 1 summarizes the comparison with sham controls of a series of biochemical variables and other characteristics from the sepsis and gut-IR groups. The mean age, body weight, total volume of maintenance fluid used, and the total volume of gut-lymph collected did not differ significantly between the groups. However, sepsis rats had frequent episodes of decreased MAP $(<40 \mathrm{mmHg}$, Tukey adjusted $P=0.003)$ and $/$ or increased body temperature $\left(>38^{\circ} \mathrm{C}\right.$, Adj . $P=0.03$ ) toward the end of the study period compared to the sham controls.

The sepsis rats after $5 \mathrm{~h}$ compared with sham had evidence of liver dysfunction (ALT, sevenfold increase, Adj. $P=0.0015$; AST, sixfold increase, $A d j . P=0.001)$ and renal dysfunction (creatinine, fourfold increase, Adj. $P=0.0003$; urea, 1.7-fold increase, $A d j . P=0.01$ ) in the plasma. Their plasma samples also had decreased albumin (1.4-fold, Adj. $P=0.03$ ), glucose (5.6-fold, Adj. $P=0.001$ ), and total protein (1.4-fold, Adj. $P=0.008)$. ALT and AST levels were also measured in their gut-lymph samples, but were not significantly different between the sepsis and sham groups. In 
Table 1 General sample characteristics, biochemistry, and lipid test results of rat study groups and samples

\begin{tabular}{|c|c|c|c|c|c|c|}
\hline & \multirow[t]{2}{*}{ Variable } & \multicolumn{3}{|c|}{ Mean of each study group } & \multicolumn{2}{|l|}{$95 \%$ confidence interval } \\
\hline & & Sham $(N=4)$ & Sepsis $(N=4)$ & Gut-IR $(N=4)$ & Sepsis versus Sham & Gut-IR versus Sham \\
\hline \multirow[t]{3}{*}{ Rat } & Age (day) & 87 & 78 & 82 & $(-20.72$ to 4.22$)$ & $(-16.72$ to 8.22$)$ \\
\hline & Body weight (g) & 438 & 471 & 450 & $(-15.08$ to 80.08$)$ & $(-35.83$ to 59.33$)$ \\
\hline & $\begin{array}{l}\text { Total volume of mainte- } \\
\text { nance fluid used (ml) }\end{array}$ & 45 & 64 & 50 & (0.93 to 36.58$)$ & $(-12.9$ to 22.76$)$ \\
\hline \multirow[t]{2}{*}{$\begin{array}{l}\text { Endpoint physiological } \\
\text { data }\end{array}$} & $\begin{array}{l}\text { Mean arterial pressure } \\
(\mathrm{mmHg})\end{array}$ & 78.5 & 34.8 & 75.8 & $(-65.27 \text { to }-22.23)^{* *}$ & $(-24.27$ to 18.77$)$ \\
\hline & Temperature $\left({ }^{\circ} \mathrm{C}\right)$ & 36.7 & 38.5 & 37.0 & $(0.45 \text { to } 3.05)^{*}$ & $(-1.03$ to 1.58$)$ \\
\hline \multirow[t]{12}{*}{ Endpoint plasma } & Albumin (g/l) & 22 & 16 & 19 & $(-10.2 \text { to }-1.8)^{*}$ & $(-7.2$ to 1.2$)$ \\
\hline & $\operatorname{ALT}(\mathrm{U} / 1)^{\mathrm{a}}$ & 47 & 353 & 78 & $(3.09 \text { to } 17.92)^{* *}$ & (0.69 to 3.97$)$ \\
\hline & $\operatorname{AST}(\mathrm{U} / 1)^{\mathrm{a}}$ & 104 & 625 & 213 & $(2.82 \text { to } 12.68)^{* *}$ & $(0.96$ to 4.33$)$ \\
\hline & Glucose $(\mathrm{mmol} / \mathrm{l})$ & 12.0 & 2.1 & 15.8 & $(-13.99 \text { to }-5.7)^{* *}$ & $(-0.36$ to 7.93$)$ \\
\hline & Creatinine $(\mu \mathrm{mol} / \mathrm{l})$ & 29.2 & 114.4 & 39.1 & (54.85 to 115.49$)^{* * *}$ & $(-20.42$ to 40.23$)$ \\
\hline & Urea (mmol/l) & 10.9 & 18.4 & 14.0 & $(2.85 \text { to } 12.06)^{*}$ & $(-1.56$ to 7.66$)$ \\
\hline & APOA1 $(\mathrm{mg} / \mathrm{l})^{\mathrm{a}}$ & 72 & 88 & 45 & $(0.26$ to 5.86$)$ & (0.13 to 2.98$)$ \\
\hline & APOB (mg/l) & 113 & 75 & 88 & $(-100.5$ to 24.5$)$ & $(-87.3$ to 37.8$)$ \\
\hline & Cholesterol (mmol/l) & 1.6 & 1.1 & 1.6 & $(-0.9$ to -0.04$)$ & $(-0.43$ to 0.43$)$ \\
\hline & $\begin{array}{l}\text { HDL-cholesterol } \\
\text { (mmol/l) }\end{array}$ & 0.98 & 0.59 & 0.90 & $(-0.58 \text { to }-0.2)^{* *}$ & $(-0.27$ to 0.12$)$ \\
\hline & Triglyceride (mmol/l) & 1.9 & 1.5 & 1.4 & $(-1.76$ to 1.01$)$ & $(-1.85$ to 0.92$)$ \\
\hline & Total protein $(\mathrm{g} / \mathrm{l})$ & 36 & 26 & 32 & $(-15.4 \text { to }-4.3)^{* *}$ & $(-10.1$ to 1.0$)$ \\
\hline \multirow[t]{9}{*}{ Gut-lymph } & $\begin{array}{l}\text { Total volume of gut- } \\
\text { lymph collected (ml) }\end{array}$ & 11.5 & 8.8 & 8.9 & $(-9.16$ to 3.76$)$ & $(-9.11$ to 3.81$)$ \\
\hline & $\operatorname{ALT}(\mathrm{U} / 1)^{\mathrm{a}}$ & 34 & 34 & 104 & $(0.45$ to 2.19$)$ & $(1.48 \text { to } 6.43)^{*}$ \\
\hline & AST (U/l) & 97 & 92 & 167 & $(-56.71$ to 47.25$)$ & $(22.07 \text { to } 118.31)^{*}$ \\
\hline & APOA1 (mg/l) & 238 & 121 & 136 & $(-208.04 \text { to }-25.29)^{*}$ & $(-186.5$ to -17.3$)$ \\
\hline & APOB (mg/l) & 129 & 134 & 157 & $(-54.82$ to 64.4$)$ & $(-27.7$ to 82.7$)$ \\
\hline & Cholesterol (mmol/l) & 0.39 & 0.39 & 0.34 & $(-0.09$ to 0.09$)$ & $(-0.13$ to 0.04$)$ \\
\hline & $\begin{array}{l}\text { HDL-cholesterol } \\
(\mathrm{mmol} / \mathrm{l})\end{array}$ & 0.03 & 0.07 & 0.03 & $(-0.006$ to 0.08$)$ & $(-0.04$ to 0.04$)$ \\
\hline & Triglyceride $(\mathrm{mmol} / \mathrm{l})$ & 2.13 & 1.68 & 1.65 & $(-1.15$ to 0.24$)$ & $(-1.13$ to 0.16$)$ \\
\hline & Total protein $(\mathrm{g} / \mathrm{l})$ & 9.1 & 6.6 & 5.9 & $(-7.7$ to 2.7$)$ & $(-8.0$ to 1.6$)$ \\
\hline \multirow[t]{6}{*}{ Gut-lymph TRL } & APOA1 (mg/l) & 321 & 184 & 214 & $(-309.1$ to 34.1$)$ & $(-279.1$ to 64.1$)$ \\
\hline & APOB (mg/l) & 116 & 144 & 150 & ( -63.3 to 119.6$)$ & $(-57.1$ to 125.8$)$ \\
\hline & Cholesterol (mmol/l) & 0.57 & 0.49 & 0.44 & $(-0.22$ to 0.06$)$ & $(-0.27$ to 0.01$)$ \\
\hline & $\begin{array}{l}\text { HDL-cholesterol } \\
\text { (mmol/l) }\end{array}$ & ND & ND & ND & & \\
\hline & Triglyceride (mmol/l) & 4.2 & 3.3 & 3.1 & $(-2.48$ to 0.73$)$ & $(-2.69$ to 0.52$)$ \\
\hline & Total protein $(\mathrm{g} / \mathrm{l})$ & ND & ND & ND & & \\
\hline
\end{tabular}

$A L T$ alanine aminotransferase, $A S T$ aspartate aminotransferase, $N$ number of samples, $N D$ not detectable

Data shown are the means and $95 \%$ confidence intervals for the pairwise differences between the means

Statistically significant Tukey adjusted $P$ values: $* P<0.05 ; * * P<0.01 ; * * * P<0.001$

${ }^{a}$ Analyses were performed on log-transformed data, and their $95 \%$ confidence intervals are for ratios of means

contrast, plasma from gut-IR rats did not have an increase in any analytes, but there was a significant increase in ALT and AST (threefold and 1.7-fold, respectively, both Adj. $P=0.02)$ in the gut-lymph compared with sham.

\section{Lipid Profile Tests}

A few commonly used lipid profile laboratory tests (TG, cholesterol, APOA1, APOB, and HDL-cholesterol) were performed for the endpoint plasma, gut-lymph, and gutlymph TRL-enriched fractions. In general, the lipid profile 
of the samples did not differ statistically between the three groups (Table 1). There were, however, some important exceptions: In the sepsis-induced rats, there was a significant decrease in plasma HDL-cholesterol (40\% decrease, Adj. $P=0.003$ ) and gut-lymph APOA1 (50\% decrease, Adj. $P=0.04$ ). Lower TG and APOA1 levels, but higher APOB levels, were measured in both gut-lymph and their TRLenriched fractions of both sepsis and gut-IR groups in comparison with the sham, but did not reach the statistical significance (Table 1). Our test results (Table 1) indicated that gut-lymph TRL-enriched fractions contained undetectable levels of total protein and HDL-cholesterol, but contained twofold higher level of TG $(P<0.0001$ by paired two-tailed $\mathrm{T}$ test) than the gut-lymph, supporting the purification of TRL in the TRL-enriched fractions. The APOB test was designed to measure the APOB100 and was not specific to the APOB48 present on the chylomicrons. The APOB test results showed similar concentrations of APOB in the gutlymph samples and their TRL-enriched fractions.

\section{Altered Non-polar Metabolites}

Analysis of non-polar metabolites by UHPLC-MS detected 190 lipid species in the gut-lymph TRL-enriched fractions, of which $20(10.5 \%)$ were unidentified (Fig. 1a). The majority of identified lipids were either glycerolipids (40\%; $76 / 190)$ or glycerophospholipids $(36 \%$; 68/190), with triacylglycerols (TG; 27\%; 52/190) and phosphocholines (PC; $24 \%$; 45/190) being the main subclasses of glycerolipids and glycerophospholipids, respectively. Many lipid species were composed of long-chain fatty acids with aliphatic tails of 18-22 carbons. Lysophosphatidylcholine (LPC) and lysophosphatidylethanolamine (LPE) are emerging as a novel class of inflammatory lipids [21] and previously found to be increased in the gut-lymph of rodent trauma and hemorrhagic shock model [22], but were not identified in any of 12 gut-lymph TRL-enriched fractions in our study. All the non-polar metabolites identified and quantified are shown in the Supplementary Data 1.

In sepsis gut-lymph TRL-enriched fractions, there were significant decreases (FDR-adjusted $P<0.05$ ) in 35/190 lipid species and no significant increases in any lipid species were observed (Fig. 1b). Of those altered lipid species, 94\% (33/35) were either glycerolipids or glycerophospholipids: 3 diacylglycerols (DG; $17.6 \%$ of total 17 DG identified), 11 TG (21.2\% of 52), 8 PC (17.8\% of 45), 5 phosphoethanolamines (PE; 71.4\% of 7), and 6 phosphoserines (PS; $46.2 \%$ of 13). While all 33 lipid species were significantly decreased
Fig. 1 Non-polar metabolite features in gut-lymph TRLenriched fractions. (a) Nonpolar metabolites identified in this study were grouped by lipid classes. (b) Percentage of non-polar metabolite features that were upregulated, downregulated, or not significantly changed in sepsis or gut-IR in comparison with the sham control rats. Only the metabolites with false discovery rate (FDR)-adjusted $P$ value $<0.05$ are considered statistically significant. All metabolites identified and quantified are shown in Supplementary Data 1. $M G$ monoacylglycerols, $D G$ diacylglycerols, $T G$ triacylglycerols, $P A$ phosphates, $P E$ phosphoethanolamines, $P I$ phosphoinositols, $P S$ phosphoserines, $P C$ phosphocholines. Note Twenty out of $190(10.5 \%)$ non-polar metabolites were unidentifiable. Results from these unidentified metabolites are reported in the Supplementary Data 1

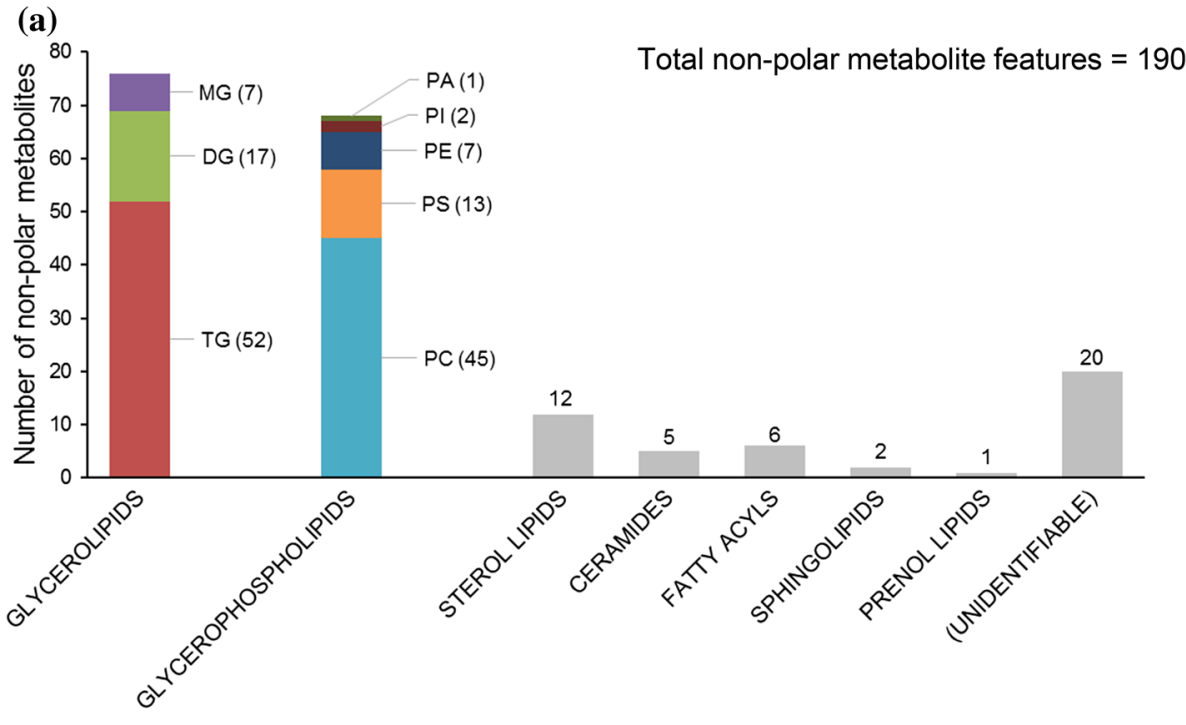

(b)

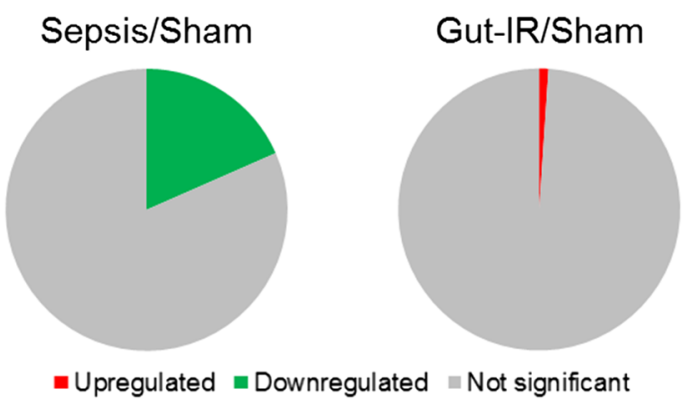


in sepsis, all decreases were less than twofold relative to sham (Figs. 2, 3).

In gut-IR gut-lymph TRL-enriched fractions, there were significant changes in only 2 lipid species (Fig. 1b). Both of these were monoacylglycerol (MG) species: $\mathrm{MG}(18: 1)$ and MG(18:2), and they both showed $\sim$ twofold increase (Adj. $P=0.045$ and $P=0.007$, respectively) in gut-IR compared with sham (Fig. 2). There was a non-significant decrease trend (all Adj. $P>0.05$; Figs. 2, 3) for many lipid species. There were no non-polar metabolites identified in this study that were significantly and similarly altered in both the sepsis and gut-IR groups.

\section{Altered Polar Metabolites}

Overall in all three rat groups, there were only 21 polar metabolites detected in the gut-lymph TRL-enriched fractions using GC-MS approach (Fig. 4). One of them was unidentifiable, and the rest were all present at low but measurable concentrations. In the TRL-enriched fractions, myo-inositol metabolite was increased in sepsis (2.6-fold increase, Adj. $P=0.03$ ) but not in gut-IR. None of the other metabolite concentrations changed significantly, and none changed by more than twofold in sepsis or gut-IR compared with sham. None of the polar metabolites identified in this study were significantly and similarly altered in both sepsis and gut-IR groups. All polar metabolites identified and quantified are listed in the Supplementary Data 1.

\section{Discussion}

TRLs are important in lipid absorption and energy distribution. TRLs are of particular relevance to the gut-lymphespecially for the chylomicrons, gut-lymph is the only anatomical route that dietary lipid in newly synthesized chylomicrons move from the gut to the systemic circulation. Gut-lymph has been identified as a source of toxic factors that promote MODS [23-25] in critical illness. Gutlymph also enters the bloodstream immediately prior to the heart and lungs, the two organs that most commonly fail in MODS. There is evidence that lipoproteins (but not limited to TRLs), which are oxidized [26, 27] or carry bioactive factors [28], can contribute to disease development. But the content of gut-lymph TRLs has not been studied in detail in critical illness nor MODS.

There have been several publications on plasma or serum metabolites in sepsis [29-41] and gut-IR [42, 43]. But we

(a)

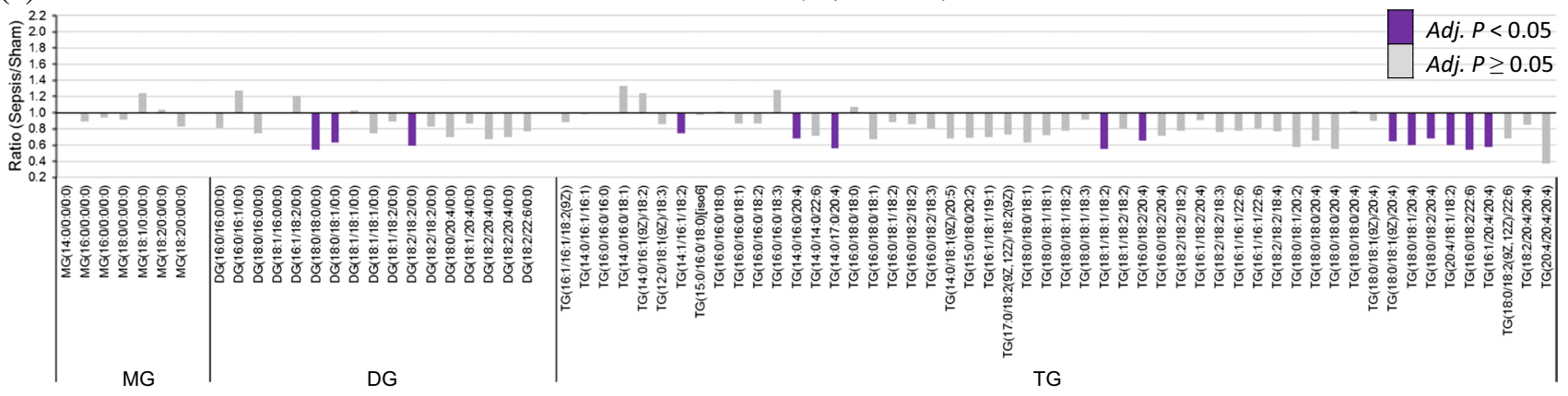

(b)

GLYCEROLIPIDS (Gut-IR/Sham)

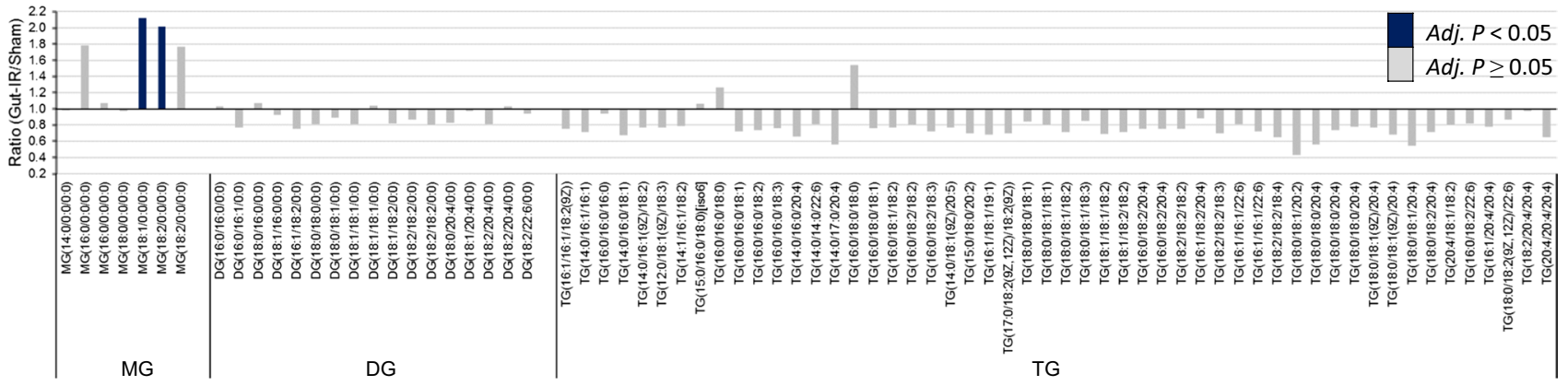

Fig. 2 Altered glycerolipid profile in the gut-lymph TRL-enriched fractions in sepsis and gut-IR. All MG, DG, and TG species identified in this study are shown with the disease/sham ratio: a sepsis/sham; b gut-IR/sham. Each bar is color-coded by the FDR-adjusted $P$ value:
$P<0.05$, significantly changed; $P \geq 0.05$, not significantly changed. Note that a same lipid ID was given to a lipid with different adducts or identified in both positive and negative modes. $M G$ monoacylglycerols, $D G$ diacylglycerols, $T G$ triacylglycerols 


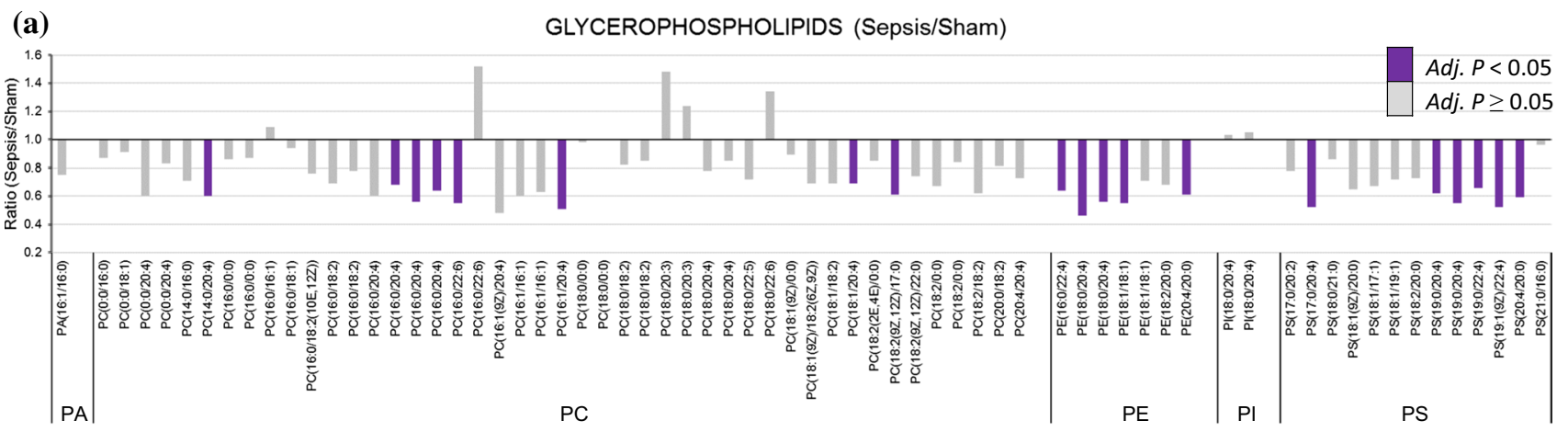

(b) GLYCEROPHOSPHOLIPIDS (Gut-IR/Sham)

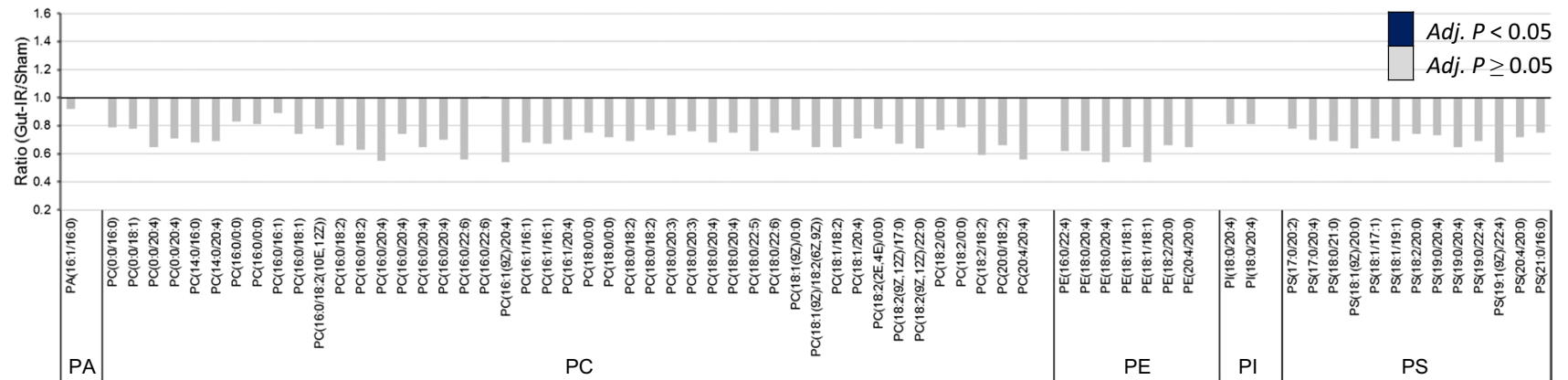

Fig. 3 Altered glycerophospholipid profile in the gut-lymph TRLenriched fractions in sepsis and gut-IR. All PA, PC, PE, PI, and PS species identified in this study are shown with the disease/sham ratio: a sepsis/sham; b gut-IR/sham. Each bar is color-coded by the FDRadjusted $P$ value: $P<0.05$, significantly changed; $P \geq 0.05$, not sig- nificantly changed. Note that a same lipid ID was given to a lipid with different adducts or identified in both positive and negative modes. $P A$ phosphates, $P C$ phosphocholines, $P E$ phosphoethanolamines, $P I$ phosphoinositols, $P S$ phosphoserines
Fig. 4 Changes in polar metabolite features in gut-lymph TRL-enriched fraction in sepsis or gut-IR in comparison with sham control rats. Disease/sham ratio and FDR-adjusted $P$ value are shown. Polar metabolites with an adjusted $P$ value $<0.05$ are considered statistically significant. Note A total of 21 polar metabolites features were detected, but one of them was unidentifiable. The unidentifiable metabolite was omitted in this figure, but presented in the Supplementary Data 1

Myo-inositol
Malic acid
L-Proline
Creatinine
2-ketoglutaric acid
Citric Acid
Pyroglutamic acid
L-phenylalanine
Urea
Octadecanoic acid
Glucose
Hexadecanoic acid
Lactic acid
L-threonic acid
Phosphoric acid
Glycerol
Beta-hydroxybutyric acid
Glycine
L-Valine
L-Alanine

\section{Sepsis/Sham}

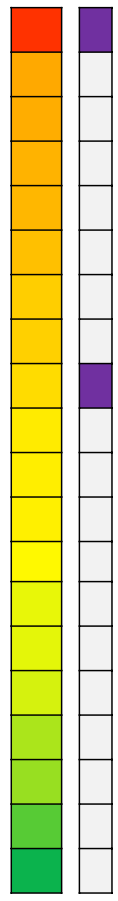

Ratio Adj. $P$ value

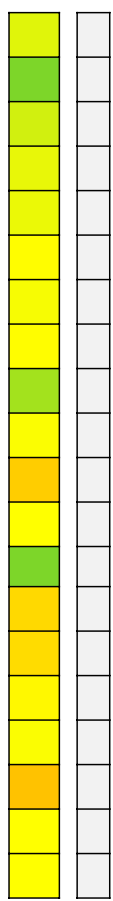

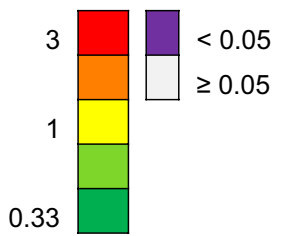


could only find one metabolite study on the gut-lymph in sepsis [44] and none on the TRL-enriched fraction of the gut-lymph. This is therefore the first study to compare polar and non-polar metabolite profiles in TRL-enriched fractions of gut-lymph in animal models of sepsis and gut-IR. Overall the gut-lymph TRL fraction showed a restricted range of polar and non-polar metabolites across all the groups. A striking feature of the study on non-polar metabolites was the discovery of a global depression in multiple lipid species of the gut-lymph TRL fraction in sepsis, and only MG(18:1) and MG(18:2) significantly increased in gut-IR (Fig. 2). We found there to be a generally low polar metabolite content in TRLs in both sepsis and gut-IR, but there was a significant increase in myo-inositol in the gut-lymph TRLs of sepsis (Fig. 4). In this study, we could not find any common metabolites (both polar and non-polar) altered in the TRL-enriched fractions of both sepsis and gut-IR.

Myo-inositol was increased in sepsis in this study. This compound is a component of cell membrane, as well as important in cell growth and survival, signal transduction cascades [45], glucose homeostasis [46], lipid metabolism [47], and osmoregulation [48]. It is also commonly found as phytate [49] in plant foodstuffs and is especially high in cereals. Dietary phytate is converted into myo-inositol in the intestine by the enzyme phytase [49] and is actively absorbed from the intestine through the $\mathrm{Na}^{+} / \mathrm{K}^{+}$-ATPase transport system [50]. Various bacterial species in the microbiome have phytate-degrading enzymes [51], and it is known that dysbiosis occurs in sepsis [52]. One mechanism by which myo-inositol might increase in gut-lymph TRLs is by an increase in bacterial phytate-degrading enzymes in the intestine. In addition to dietary sources, myo-inositol is also synthesized from glucose in various organs [45], and glycemic dysregulation is a key feature of critical illness, in particular within patients with sepsis [53, 54]. Increased serum/plasma and tissue myo-inositol levels have been demonstrated in earlier studies of sepsis [39-41], but never in gut-lymph. In septic rats, Izquierdo-García et al. [39] reported increased concentrations of myo-inositol within lung tissue. In patients, Mickiewicz et al. [40] reported increased myo-inositol in serum during septic shock, and Stringer et al. [41] reported the positive association between the plasma levels of myo-inositol and disease severity (acute physiology score) in sepsis-induced acute lung injury. Supported by the anatomical route of gut-lymph and a previous study that demonstrated an uptake of myo-inositol into the lung cells [55], the increased availability of myo-inositol in gut-lymph may also raise its levels in the lungs. In addition, myo-inositol exists either in its free form, phosphate derivatives, or phospholipid-bound form [45], and small but significant amounts of the phospholipid-bound form are known to be associated with serum lipoproteins [56]. Thus, the measured levels of myo-inositol would be affected by any factors that can alter the ratio between different myo-inositol forms. Increased myo-inositol levels could be involved in cell biology related to sepsis pathogenesis. Given these findings from our study and others, a more systematic study of the role of myo-inositol in sepsis is warranted.

Although the majority of non-polar metabolites were unchanged, there was an increase in $\operatorname{MG}(18: 1)$ and MG(18:2) in the gut-lymph TRL fractions in the rodent models of gut-IR (Fig. 2). There are a few possible explanations for this. Although TGs typically reform within the enterocytes from the absorbed fatty acids and MG before being incorporated into chylomicrons, some MGs may be incorrectly incorporated into TRLs when the gut barrier function is damaged from gut-IR. While MGs could have been produced, respectively, via enzymatic hydrolysis of TGs and DGs by lipoprotein lipase [57-59] and diacylglycerol lipase [59] during collection of gut-lymph, MGs would not likely be associated with TRLs in this situation. The increase in MGs in TRL fractions was only observed in gut-IR and not sepsis, and this occurred when most of the other lipid species showed a decreasing trend. As such, it is worth evaluating MGs as a potential biomarkers of intestinal injury.

Comparing two animal models of critical illness provided the opportunity to identify changes in metabolite profile that were common to both, which would have suggested a common TRL-mediated mechanistic pathway in the development of MODS. Although this was not observed, it was notable that there were significant decreases in 35 lipid species in sepsis and a decreasing trend in almost all lipid species in gut-IR gut-lymph TRLs (Figs. 2, 3). This strongly suggests that critical illness is associated with a decrease in most lipid species in gut-lymph TRLs. This is also supported by the finding that total TG is decreased in gut-lymph TRL fractions in both animal models (Table 1). The reduction appears to be independent of confounding factors such as hemodilution since the groups were not significantly different for total fluid administered. In addition, there were no biochemical signs of hemodilution (e.g., lower urea or creatinine) in the sepsis or gut-IR groups.

The decrease in lipid species associated with gut-lymph TRLs in both animal models was not thought to be due to other changes with the TRLs. Direct Detect assay, nanoparticle tracking analysis, and protein PAGE performed on the gut-lymph TRL-enriched fractions showed no difference in the protein concentration, particle concentrations and sizes, and protein banding patterns, respectively, between the study groups (Supplementary Data 2). This means that similar numbers and sizes of TRL particles with conserved major protein content are produced by the intestine and carried by the gut-lymph in these animal models. However, what was consistent was that the incorporation of lipid into the TRLs appears to be decreased in these diseases. Since the 
rat surgery, metabolite extraction, and sample injection into the instrument for MS analyses were randomized, the specific decrease in lipid content in the disease groups relative to sham is likely due to disease state rather than being an artifact of collection or hemodilution.

It is tempting to speculate that this decreased carriage of lipids in the gut-lymph TRLs may have wider implications for nutrition during critical illness. Lipid is the main content of TRLs, and the TGs are their major lipid components [8]. Less TG content in the gut-lymph TRLs could decrease energy supply to the body and impact the vital organ function during critical illness. Metabolic alterations, abnormal fuel utilization, and failure to meet the energy requirements of peripheral tissues have been noted in several studies of critical illness and sepsis [60-63]. Our finding may therefore represent supporting evidence for an early change in TRL lipid carriage that could underpin these clinical observations.

To our knowledge, there has been only one study [22] that profiled the metabolome in whole gut-lymph using mass spectrometry. In a rodent model of trauma and hemorrhagic shock, Morishita et al. [22] found on lipidomic analysis that LPC and LPE with polyunsaturated fatty acids were present in gut-lymph and probably contributed to acute lung injury and MODS. In the present study, we analyzed the TRLenriched fractions of gut-lymph, and the LPC and LPE identified as biologically active lipids by Morishita et al. were not detected. In fact, TRLs of gut-lymph are not expected to contain much lysophospholipids such as LPC and LPE. Phospholipids, which are predominant dietary lipids along with TGs, are hydrolyzed to lysophospholipids (primarily LPC) in the intestinal lumen and absorbed into enterocytes. But then those lysophospholipids are either 1) re-esterified to phospholipids and assembled into chylomicrons before released into lacteals; or 2) transported to the plasma directly and promote systemic inflammation and cell dysfunction [21]. We suspect that those lipids identified in Morishita et al.'s study might be unique trauma-specific lipids, present in the gut-lymph at very low abundance, or not carried in the TRLs.

There are some acknowledged limitations in this first exploratory study of TRL composition in critical illness. Firstly, any one rodent model does not necessarily reflect what happens in patients with a critical illness. Recognizing this, we elected to study two different models. "Sepsis" is one of the most common causes of critical illness and admission to intensive care units [64], and the model we used consistently produces MODS. Hypoperfusion of the gut is a feature in critical illnesses so is a suitable second model. However, that too has limitations as the mechanism for gut-IR in our model is not reflex splanchnic vasoconstriction that occurs in critical illness to maintain vital organ perfusion but rather was temporary occlusion of the superior mesenteric artery. Secondly, the use of
TRL-enriched fractions, which combined both chylomicrons and VLDL, prevented the identification of specific changes in the metabolome of either. During the $5 \mathrm{~h}$ after disease induction, the rats inevitably had a gradual fed to fasted transition which meant there were likely to produce more chylomicrons initially and relatively more VLDLs later. We decided that the inclusion of both classes of lipoproteins was the best strategy for this exploratory study and probably more relevant to the abnormal physiology of critical illness. Thirdly, the study design prevented the identification of amphiphilic lipids because only the nonpolar phase extract was used for the UHPLC-MS analysis. This class of lipids will require a dedicated study with a larger sample volume. Finally, there is the generic constraint with lipidomics studies that all lipids cannot be identified because similar mass and structures, or the inability to unequivocally assign a chemical structure. The lipidomics data analysis workflow in this study produced almost 200 putative non-polar metabolites. Metabolites identified by this workflow are considered to be correct for lipid class and size, but there were limitations in some cases beyond this level of resolution. The chain combinations known to be more prevalent were assigned in preference over the less common options (for example, 16:0/18:0 was assigned rather than 17:0/17:0); and an identical metabolite identification was given to more than one metabolite if they were detected in both positive and negative ion modes, or with different adducts.

This exploratory study has been the first to investigate the metabolome of TRL-enriched fractions in gut-lymph. We have developed several hypotheses for future testing and confirmation. We found significantly increased levels of myo-inositol and two MGs in rodent models of sepsis and gut-IR, respectively. There was also a striking decrease in levels of many lipid species in the TRL-enriched fractions in sepsis and to a lesser extent in gut-IR. These findings provide the basis for a series of new studies to confirm these profile changes in clinical samples. They also support new investigations into how the metabolomic profile of lymph components changes in critical illness after fat content modulation of the diet or alterations to the resident microbiome through antibiotics or substrate manipulation.

Acknowledgments We wish to thank C Keven of the Liggins Institute, University of Auckland, for the biochemical analyte measurements; S Church and P Begley from the Centre for Advanced Discovery and Experimental Therapeutics, Division of Cardiovascular Sciences, Faculty of Biology, Medicine and Health at the University of Manchester, UK (supported by the NIHR Manchester Biomedical Research Centre), for their technical assistance with the metabolomic analyses.

Author's contributions JH, ARP, AJH, and JAW designed the studies. $\mathrm{SN}, \mathrm{SMT}$, and AP performed animal surgery. JH interpreted the data and wrote the manuscript, and ARP, AJH, and JAW edited the manuscript. All authors approved the manuscript. 
Funding This work was supported by the Early Careers Academic Grants, Association of Commonwealth Universities; Maurice and Phyllis Paykel Trust; Performance-Based Research Fund, University of Auckland; Auckland Medical Research Foundation; Faculty Research Development Fund, University of Auckland; and Health Research Council of New Zealand.

\section{Compliance with ethical standards}

Conflict of interest The authors declare that they have no conflict of interest.

Ethical approval All applicable international, national, and/or institutional guidelines for the care and use of animals were followed. All procedures performed in studies involving animals were in accordance with the ethical standards of the institution or practice at which the studies were conducted.

\section{References}

1. Urden LD, Stacy KM, Lough ME. Critical Care Nursing: Diagnosis and Management. 7th ed. Amsterdam: Elsevier; 2014.

2. Dewar D, Moore FA, Moore EE, Balogh Z. Postinjury multiple organ failure. Injury. 2009;40:912-918.

3. Swank GM, Deitch EA. Role of the gut in multiple organ failure: bacterial translocation and permeability changes. World J Surg. 1996;20:411-417.

4. Deitch EA, Xu D, Kaise VL. Role of the gut in the development of injury- and shock induced SIRS and MODS: the gut-lymph hypothesis, a review. Front Biosci. 2006;11:520-528.

5. Bisgaier CL, Glickman RM. Intestinal synthesis, secretion, and transport of lipoproteins. Annu Rev Physiol. 1983;45:625-636.

6. Ockner RK, Hughes FB, Isselbacher KJ. Very low density lipoproteins in intestinal lymph: origin, composition, and role in lipid transport in the fasting state. J Clin Invest. 1969;48:2079-2088.

7. Green PH, Glickman RM, Saudek CD, Blum CB, Tall AR. Human intestinal lipoproteins. Studies in chyluric subjects. J Clin Invest. 1979;64:233-242.

8. Jonas A, Phillips MC. Lipoprotein structure. In: Vance DE, ed. Biochemistry of Lipids, Lipoproteins and Membranes. 5th ed. Vance JE: Elsevier B.V; 2008:485-506.

9. Lo CM, Nordskog BK, Nauli AM, et al. Why does the gut choose apolipoprotein B48 but not B100 for chylomicron formation? Am J Physiol Gastrointest Liver Physiol. 2008;294:G344-G352.

10. Ali Abdelhamid Y, Cousins CE, Sim JA, et al. Effect of critical illness on triglyceride absorption. JPEN J Parenter Enteral Nutr. 2015;39:966-972.

11. Lind L, Lithell H. Impaired glucose and lipid metabolism seen in intensive care patients is related to severity of illness and survival. Clin Intensive Care. 1994;5:100-105.

12. Wishart DS, Knox C, Guo AC, et al. HMDB: a knowledgebase for the human metabolome. Nucleic Acids Res. 2009;37:D603-D610.

13. Otero-Anton E, Gonzalez-Quintela A, Lopez-Soto A, et al. Cecal ligation and puncture as a model of sepsis in the rat: influence of the puncture size on mortality, bacteremia, endotoxemia and tumor necrosis factor alpha levels. Eur Surg Res. 2001;33:77-79.

14. Akcakaya A, Alimoglu O, Sahin M, Abbasoglu SD. Ischemiareperfusion injury following superior mesenteric artery occlusion and strangulation obstruction. J Surg Res. 2002;108:39-43.

15. Windmueller HG, Levy RI. Production of beta-lipoprotein by intestine in the rat. J Biol Chem. 1968;243:4878-4884.
16. Cox RA, García-Palmieri MR. Cholesterol, Triglycerides, and Associated Lipoproteins. Boston: Butterworth Publishers; 1990.

17. Oikawa S, Mizunuma Y, Iwasaki Y, Tharwat M. Changes of very low-density lipoprotein concentration in hepatic blood from cows with fasting-induced hepatic lipidosis. Can J Vet Res. 2010;74:317-320.

18. R: A language and environment for statistical computing. $\mathrm{R}$ Foundation for Statistical Computing, Vienna, Austria. http:// www.R-project.org/

19. Dunn WB, Broadhurst D, Begley P, et al. Procedures for largescale metabolic profiling of serum and plasma using gas chromatography and liquid chromatography coupled to mass spectrometry. Nat Protoc. 2011;6:1060-1083.

20. Begley P, Francis-McIntyre S, Dunn WB, et al. Development and performance of a gas chromatography-time-of-flight mass spectrometry analysis for large-scale nontargeted metabolomic studies of human serum. Anal Chem. 2009;81:7038-7046.

21. Hui DY. Intestinal phospholipid and lysophospholipid metabolism in cardiometabolic disease. Curr Opin Lipidol. 2016;27:507-512.

22. Morishita K, Aiboshi J, Kobayashi T, et al. Lipidomics analysis of mesenteric lymph after trauma and hemorrhagic shock. $J$ Trauma Acute Care Surg. 2012;72:1541-1547.

23. Flint RS, Phillips AR, Power SE, et al. Acute pancreatitis severity is exacerbated by intestinal ischemia-reperfusion conditioned mesenteric lymph. Surgery. 2008;143:404-413.

24. Deitch EA. Gut lymph and lymphatics: a source of factors leading to organ injury and dysfunction. Ann N Y Acad Sci. 2010;1207:E103-E111.

25. Qin X, Dong W, Sharpe SM, et al. Role of lipase-generated free fatty acids in converting mesenteric lymph from a noncytotoxic to a cytotoxic fluid. Am J Physiol Gastrointest Liver Physiol. 2012;303:G969-G978.

26. Lavy A, Ben Amotz A, Aviram M. Increased susceptibility to undergo lipid peroxidation of chylomicrons and low-density lipoprotein in celiac disease. Ann Nutr Metab. 1993;37:68-74.

27. Khan M, Pelengaris S, Cooper M, et al. Oxidised lipoproteins may promote inflammation through the selective delay of engulfment but not binding of apoptotic cells by macrophages. Atherosclerosis. 2003;171:21-29.

28. Sachinidis A, Kettenhofen R, Seewald S, et al. Evidence that lipoproteins are carriers of bioactive factors. Arterioscler Thromb Vasc Biol. 1999;19:2412-2421.

29. Kauppi AM, Edin A, Ziegler I, et al. Metabolites in blood for prediction of bacteremic sepsis in the emergency room. PLoS ONE. 2016;11:e0147670.

30. Beloborodova NV, Olenin AY, Pautova AK. Metabolomic findings in sepsis as a damage of host-microbial metabolism integration. J Crit Care. 2018;43:246-255.

31. Park DW, Kwak DS, Park YY, et al. Impact of serial measurements of lysophosphatidylcholine on 28-day mortality prediction in patients admitted to the intensive care unit with severe sepsis or septic shock. J Crit Care. 2014;29:882 e885-811.

32. Ferrario M, Cambiaghi A, Brunelli L, et al. Mortality prediction in patients with severe septic shock: a pilot study using a target metabolomics approach. Sci Rep. 2016;6:20391.

33. Cambiaghi A, Pinto BB, Brunelli L, et al. Characterization of a metabolomic profile associated with responsiveness to therapy in the acute phase of septic shock. Sci Rep. 2017;7:9748.

34. Evangelatos N, Bauer P, Reumann M, et al. Metabolomics in sepsis and its impact on public health. Public Health Genom. 2017;20:274-285.

35. Su L, Huang Y, Zhu Y, et al. Discrimination of sepsis stage metabolic profiles with an LC/MS-MS-based metabolomics approach. BMJ Open Respir Res. 2014;1:e00056. 
36. Eckerle M, Ambroggio L, Puskarich MA, et al. Metabolomics as a driver in advancing precision medicine in sepsis. Pharmacotherapy. 2017;37:1023-1032.

37. Ludwig KR, Hummon AB. Mass spectrometry for the discovery of biomarkers of sepsis. Mol Biosyst. 2017;13:648-664.

38. Mickiewicz B, Vogel HJ, Wong HR, Winston BW. Metabolomics as a novel approach for early diagnosis of pediatric septic shock and its mortality. Am J Respir Crit Care Med. 2013;187:967-976.

39. Izquierdo-Garcia JL, Nin N, Ruiz-Cabello J, et al. A metabolomic approach for diagnosis of experimental sepsis. Intensive Care Med. 2011;37:2023-2032.

40. Mickiewicz B, Tam P, Jenne CN, et al. Integration of metabolic and inflammatory mediator profiles as a potential prognostic approach for septic shock in the intensive care unit. Crit Care. 2015;19:11.

41. Stringer KA, Serkova NJ, Karnovsky A, et al. Metabolic consequences of sepsis-induced acute lung injury revealed by plasma $\mathrm{H}$-1-nuclear magnetic resonance quantitative metabolomics and computational analysis. Am J Physiol Lung Cell Mol Physiol. 2011;300:L4-L11.

42. Fahrner R, Beyoglu D, Beldi G, Idle JR. Metabolomic markers for intestinal ischemia in a mouse model. J Surg Res. 2012;178:879-887.

43. Khadaroo RG, Churchill TA, Tso V, et al. Metabolomic profiling to characterize acute intestinal ischemia/reperfusion injury. PLoS ONE. 2017;12:e0179326.

44. Li Y, Hou M, Wang JG, et al. Changes of lymph metabolites in a rat model of sepsis induced by cecal ligation and puncture. $J$ Trauma Acute Care Surg. 2012;73:1545-1552.

45. Holub BJ. Metabolism and function of myo-inositol and inositol phospholipids. Annu Rev Nutr. 1986;6:563-597.

46. Croze ML, Soulage CO. Potential role and therapeutic interests of myo-inositol in metabolic diseases. Biochimie. 2013;95:1811-1827.

47. Hayashi E, Maeda T, Tomita T. The effect of myo-inositol deficiency on lipid metabolism in rats. I. The alteration of lipid metabolism in myo-inositol deficient rats. Biochim Biophys Acta. 1974;360:134-145.

48. Nakanishi T, Burg MB. Osmoregulatory fluxes of myo-inositol and betaine in renal cells. Am J Physiol. 1989;257:C964-C970.

49. Holub BJ. The nutritional significance, metabolism, and function of myo-inositol and phosphatidylinositol in health and disease. Adv Nutr Res. 1982;4:107-141.
50. Caspary WF, Crane RK. Active transport of myo-inositol and its relation to the sugar transport system in hamster small intestine. Biochim Biophys Acta. 1970;203:308-316.

51. Konietzny U, Greiner R. Molecular and catalytic properties of phytate-degrading enzymes (phytases). Int J Food Sci Technol. 2002;37:791-812.

52. Haak BW, Wiersinga WJ. The role of the gut microbiota in sepsis. Lancet Gastroenterol Hepatol. 2017;2:135-143.

53. Van Cromphaut SJ, Vanhorebeek I, Van den Berghe G. Glucose metabolism and insulin resistance in sepsis. Curr Pharm Des. 2008;14:1887-1899.

54. Krinsley JS. The severity of sepsis: yet another factor influencing glycemic control. Crit Care. 2008;12:194.

55. Hallman M, Slivka S, Wozniak P, Sills J. Perinatal development of myoinositol uptake into lung cells: surfactant phosphatidylglycerol and phosphatidylinositol synthesis in the rabbit. Pediatr Res. 1986;20:179-185.

56. Breckenridge WC, Palmer FB. Fatty acid composition of human plasma lipoprotein phosphatidylinositols. Biochim Biophys Acta. 1982;712:707-711.

57. Reiser R, Bryson MJ, Carr MJ, Kuiken KA. The intestinal absorption of triglycerides. J Biol Chem. 1952;194:131-138.

58. Foster DM, Berman M. Hydrolysis of rat chylomicron acylglycerols: a kinetic model. J Lipid Res. 1981;22:506-513.

59. Young SG, Zechner R. Biochemistry and pathophysiology of intravascular and intracellular lipolysis. Genes Dev. 2013;27:459-484.

60. Kazda A. Meeting the energy requirements in sepsis. Czech Med. 1988;11:1-9.

61. Clowes GH Jr, O’Donnell TF Jr, Ryan NT, Blackburn GL. Energy metabolism in sepsis: treatment based on different patterns in shock and high output stage. Ann Surg. 1974;179:684-696.

62. Hill GL. Implications of critical illness, injury, and sepsis on lean body mass and nutritional needs. Nutrition. 1998;14:557-558.

63. Chiolero R, Revelly JP, Tappy L. Energy metabolism in sepsis and injury. Nutrition. 1997;13:45S-51S.

64. Adhikari NK, Fowler RA, Bhagwanjee S, Rubenfeld GD. Critical care and the global burden of critical illness in adults. Lancet. 2010;376:1339-1346. 